\title{
Breastfeeding and obesity: a meta-analysis
}

\author{
Jeanne M. Stolzer \\ University of Nebraska-Kearney, Kearney, USA; stolzerjm@unk.edu \\ Received 3 September 2011; revised 15 October 2011; accepted 24 October 2011.
}

\begin{abstract}
Over the last decade, obesity rates have reached epidemic proportions in the United States of America. Comorbidities associated with overweight and obesity include, but are not limited to, hypertension, type 2 diabetes, cardiovascular disease, and elevated cholesterol levels. As a direct result of obesity, data indicates that these diseases are now being detected in an unprecedented number of American children, adolescents, and adults. Although the major cause of the obesity epidemic in America has thus far been attributed to excessive caloric intake and lack of physical activity, this paper will explore the pivotal role that breastfeeding plays in the prevention of overweight and obesity throughout the life course. Epidemiological data demonstrates that breastfeeding significantly reduces the incidence of overweight and obesity and that exclusive and long term breastfeeding has been strongly correlated with a reduction in LDL cholesterol, blood pressure related disorders, type 2 diabetes, and cardiovascular dysfunction. While it is certain that diet and exercise are integral factors associated with overweight and obesity, the time has come for a collective recognition of the protective effects associated with breastfeeding if we are serious in our endeavor to eradicate the overweight and obesity epidemic in America.
\end{abstract}

Keywords: Obesity; Breastfeeding and Obesity; Breastfeeding; Lactation and Obesity

\section{INTRODUCTION}

Over the last 40 - 50 years, much attention has been given to the role of diet and exercise with regard to the prevention of obesity. It is certainly a fact that the American diet has been altered dramatically in a relatively short time. Portion sizes in food outlets have more than doubled over the last two decades, and Americans have significantly increased their consumption of fatty fast foods [1]. In addition, trans-fats are now a common ingredient in a variety of foods and natural home prepared meals are becoming increasingly rare.

In addition, Americans are now more sedentary than at any time in recorded history. Walking, which has been an integral feature of hominid existence throughout evolutionary time, has now been replaced by riding in cars, elevators, and other automated means of transportation [2]. Children no longer spend their days engaged in rigorous physical outdoor activity, but instead rely on hightech gadgetry to occupy their time [3]. Physical education classes have been systematically reduced or eliminated, and recess is no longer considered a necessary component of the American public school curriculum $[3,4]$.

According to the American Academy of Pediatrics [5], prevention of obesity in children must be the first line of defense. The American Academy of Pediatrics [5] released a policy statement that recommended that pediatricians should become adept at recognizing children at risk of overweight and obesity. In addition, pediatricians should calculate and plot Body Mass Index (BMI) at every visit, use changes in BMI to identify excessive weight gain, and monitor for comorbidities associated with obesity. The American Academy of Pediatrics [5] also states that pediatricians should encourage, support, and protect breastfeeding in order to significantly decrease overweight and obesity in child populations [2,5].

It is interesting to note that at the same time the American Academy of Pediatrics (AAP) [5] is promoting the multifarious benefits of breastfeeding, they are also aligned economically with the formula industry. The AAP [5] routinely advertises formula in the prestigeious medical journal "Pediatrics," was granted three million dollars by the manufacturers of formula to build the AAP headquarters in Illinois, and is funded in part by block grants provided by the formula industry $[6,7]$.

If the AAP [5] is sincere in its efforts to promote breastfeeding as a means to combat overweight and obesity, perhaps the time has come to sever its economic ties with the formula industry. Margolis [8] forcefully 
reiterates this position by stating "The acceptance of gifts in virtually any form violates the fundamental duties of the physician of nonmaleficence, fidelity, justice, and self improvement; the medical community must articulate this position clearly, and it should act accordingly” (p. 51).

Clearly, the prevention of this epidemic should be our focus as data confirms that a high body mass index (BMI) in childhood is a strong predictor of overweight and obesity during adolescence and adulthood [9]. Increasing breastfeeding initiation and duration will, according to published data, significantly decrease overweight and obesity, thus decreasing morbidity and mortality rates across the life span [10,11]. Obesity related diseases such as cardiovascular disease, high blood pressure, elevated cholesterol, and type 2 diabetes are manifested in both children and adults, therefore, early intervention is critical [5,12].

\section{BENEFITS OF BREASTFEEDING}

In keeping with the guidelines set forth by the Surgeon General of the United States [13] and the World Health Organization [11], infants should be exclusively breastfed for the first six months of life, with continued breastfeeding for two years or longer. According to decades of empirical, epidemiological data, breastfeeding has been associated with significant decreases in morbidity and mortality rates across the life course [13]. Breastfeeding has also been correlated with a reduction of infectious and noninfectious diseases, diarrhea, respiratory illness, ear infection, type 1 and 2 diabetes, celiac disease, inflammatory bowel disease, childhood cancer, allergies, asthma, overweight, and obesity [14,15].

Numerous scientific studies have confirmed that children who are breastfed exclusively and long term are less likely to develop botulism, bacterial meningitis, urinary tract infection, liver disease, and sudden infant death syndrome (SIDS) $[14,16]$. Furthermore, researchers have found that breastfeeding significantly decreases hospital admission rates and prescription drug use in pediatric populations [14,17].

\section{BREASTFEEDING AND OBESITY: EPIDEMIOLOGICAL EVIDENCE}

For over forty years, epidemiological studies have demonstrated that breastfeeding significantly reduces both overweight and obesity in child, adolescent, and adult populations [18-20]. In addition, empirical data has confirmed that nutritional intake in infancy is highly correlated with later predisposition to diseases such as obesity, high blood pressure, heart disease, and type 2 diabetes [21,22].
It has been established that cow milk contains signifycantly higher levels of protein and fat than human milk. The high fat and protein levels found in bovine based formula lead to an increased secretion of IGF-1 (insulin growth factor type 1 and in turn stimulates the overproduction of adipocytes which has been associated with overweight and obesity in human populations [23]. Human milk is compositionally distinct from all other mammalian milk as milk from a particular mammal is species specific (i.e., ensures the optimal development of that particular mammal). Cow milk contains three times the amount of protein that human milk contains, as the survival of most mammals is dependent on the rapid acceleration of physical growth. However, humans are distinct from most mammals in this respect [24].

Human milk is quantitatively different than formula regardless if it is soy or bovine based. Numerous bioactive factors are exclusive to human milk, including specific human growth hormones and growth factors which impact differentiation, growth, and functional maturation of the human organism $[25,26]$.

The concept that early nutritional intake influences adult onset overweight and obesity was first developed by McCance [20] in the 1960's. Kramer [18] continued on with McCance's seminal work and demonstrated with empirical case-controlled studies that formula feeding in infancy was significantly linked to obesity in adolescence and adulthood. Animal studies have also been valuable as they show the particular mechanisms that link early nutrition with accelerated growth, appetite regulation, and the underlying hormonal factors which have been correlated with overweight and obesity in human subjects [27].

It has been suggested that one of the reasons that formula-fed infants are more likely to develop overweight and obesity later in life is that there are predetermined amounts of formula that a baby is supposed to drink. Physicians and/or the manufacturers of formula recommend specific dosages which often times leads to overeating and the inability to determine satiety [25]. Conversely, a breastfed baby has no predetermined amount they are supposed to drink at each feeding. Across mammalian species, the exclusively breastfed baby suckles until satisfied. There is no overfeeding and the infant learns appetite regulation beginning immediately after birth [28].

Longitudinal data demonstrates that breastfeeding acts as a buffer against overweight and obesity even when controlling for confounding variables such as socioeconomic status, education of parents, race, number of siblings, and maternal BMI [29,30]. Gillman and Colleagues [31] found that the effects of breastfeeding are dose-response specific, as their data indicates that the 
longer a child is breastfed, the less likely the child is to become overweight or obese in later life.

Armstrong \& Reilly [29] assessed 32, 2000 children and found the most statistically powerful results were detected in exclusively breastfed populations. Those subjects who were formula fed in infancy were signifycantly more likely to develop overweight and obesity during later childhood. These results are critical as they demonstrate the protective effects of exclusive breastfeeding as opposed to formula feeding or breastfeeding using formula supplementation.

Bergman, et al. [32] conducted a longitudinal birth cohort study that tested whether exclusive breastfeeding was correlated with BMI at six years of age. They looked at newborns with almost identical BMI's, and revisited these infants at three months of age, and at four, five, and six years of age. At every interval, the formula fed children had significantly higher BMI's and thicker skin folds than the breastfed cohort. At the ages of four through six, the prevalence of obesity tripled in the formula fed population.

Other researchers have found that breastfeeding significantly decreases overweight and obesity in childhood regardless of maternal diabetes status, or weight status [33]. Gillman and Colleagues [31] work demonstrates that overweight during adolescence predicts short and long term morbidity as well as obesity in adulthood. According to Gillman, et al, formula feeding in infancy is a significant predictor of adult overweight and obesity even when controlling for variables such as gender, energy intake, time watching TV, physical activity, mother's BMI, and socioeconomic status.

Decades of systematic reviews of epidemiological studies (cohort, case-control, cross-sectional, and longitudinal studies) have compared breastfed and formula infants. These studies have adjusted for a multitude of confounding variables such as gender, race, age, maternal status, parental education, birthweight, and geographical location. All of these studies have concluded that breastfeeding plays a significant role in reducing overweight and obesity in child, adolescent, and adult populations $[18,32,33]$. Additional research have clearly demonstrated the dose-response specifity of breastfeeding as data confirms that the longer a child is breastfed, the stronger the protective effect of breastfeeding against overweight and obesity throughout life $[31,34]$.

\section{COMORBIDITIES ASSOCIATED WITH OVERWEIGHT AND OBESITY}

Numerous studies have concluded that breastfeeding not only decreases the prevalence of overweight and obesity, but is also inversely related to the development of type 2 diabetes, elevated blood pressure and choles- terol, and cardiovascular disease.

\section{TYPE 2 DIABETES}

The rates of type 2 diabetes are higher now then at any other time in recorded American history [2]. Overweight and obesity are thought to precipitate type 2 diabetes by interfering with insulin signaling as excess fat in the muscle cells leads to physiological atrophy resulting in hyperglycemia and chronic glucose intolerance [35]. Exclusive breastfeeding has been found to protect individuals from type 2 diabetes by encouraging optimal lipoprotein metabolism and gastrointestinal adaptation [2].

Exclusive breastfeeding has also been correlated with lower fasting insulin concentrations and lowered preprandial blood glucose levels in adults [36]. Ravelli [37] studied a cohort of subjects that were born between 1943 and 1947 in Amsterdam. Ravelli's data revealed that those adults who were exclusively breastfed were significantly less likely to be diagnosed with type 2 diabetes when compared to those subjects who were partially or exclusively formula fed.

Although investigations are still ongoing, there exists substantial evidence to support the supposition that breastfeeding acts as a buffer with regard to the development of type 2 diabetes in child, adolescent, and adult populations [5,9,13,15,36].

\section{ELEVATED BLOOD PRESSURE AND CHOLESTEROL}

According to the World Health Organization [15], elevated blood pressure in adolescence and adulthood has been correlated with formula feeding in infancy. Martin and Colleagues [38] conducted a systematic review which included over 17,000 adults and found that systolic blood pressure was significantly lower in subjects who were exclusively breastfed during infancy. Martin, et al. [38] acknowledged that the particular mechanisms underlying these findings are not fully understood at this time, and that more research is needed in this area.

Plagemann \& Harder [39] found that breastfeeding positively impacts both HDL and LDL cholesterol levels and significantly reduces blood pressure in adolescents and adults. Waterland \& Garza [22] hypothesized that the metabolic imprinting that occurs with formula feeding actually alters vascularization and cell structure causing changes in the production of enzymes, hormones, and transmembrane transporters. Waterland \& Garza [22] suggest that this metabolic alteration significantly increases the risks of later cardiovascular disease.

Although numerous studies have indicated that exclu- 
sive breastfeeding has been associated with a protective effect against elevated blood pressure and elevated cholesterol levels later in life [40], researchers have acknowledged that the mechanisms which regulate lipoprotein concentrations and blood pressure functioning need further investigation. What is certain at this point is that breastfeeding is statistically related to the prevention of metabolic atrophy in human populations $[15,25,40]$.

\section{CARDIOVASCULAR ATROPHY}

A number of studies have indicated that elevated BMI is correlated with cardiovascular disease [5,9,15,35], and that childhood overweight and obesity are significant predictors of later cardiovascular, dysfunction [2,41,2].

Published data indicates that formula feeding increases the risks of later cardiovascular disease. Rich-Edwards, et al., [42] examined over 87,000 individuals observing differences in feeding methods and later cardiovascular malfunction. Participants were born between 1921 and 1946 and reported in 1992 if they were breastfed or formula fed, and for how long they were breastfed. During eight years of investigation, these researchers found that breastfed populations had significantly lower rates of cardiovascular disease and stroke. The most significant protective effects of breastfeeding were observed in those participants who were breastfed for nine months or longer.

Owen, Whincup, Odoki, Gilg, and Cook [43] cross sectional study suggests that breastfeeding plays a major role in the prevention of cardiovascular disease. According to this study, breastfeeding is associated with lower LDL cholesterol and blood pressure, and has long term benefits for cardiovascular functioning.

The widely known Muscatine Study demonstrated that cardiovascular risk in adulthood is related to childhood LDL cholesterol levels and childhood BMI [41]. In addition, postmortem studies have indicated that with regard to atherosclerosis, the extent and severity of cardiovascular disease is highly correlated with elevated BMI and lipoprotein levels [2].

Researchers have postulated that infant nutrition is a reliable predictor of later cardiovascular functioning. Tracy, Newman, Wattigney, and Berenson [44] concluded that atrophy of the arterial wall most likely occurs during childhood as data indicates that formula feeding in infancy is a more powerful predictor of cardiovascular disease then is adult risk factors.

\section{CONCLUSIONS}

It has been well established that breastfeeding signifycantly decreases overweight and obesity and those comorbidities associated with these conditions $[5,15]$. How- ever, any discussion regarding increasing breastfeeding rates in America must take into account the multifarious variables associated with this issue. In order to increase breastfeeding rates across diverse populations, researchers must acknowledge the complexities associated with breastfeeding, which include, but are not limited to 1) The lack of breastfeeding role models in the family, the community, and the mass media [28]; 2) federal policies which impede both the initiation and duration of breastfeeding [6,17]; 3) culture ideologies which dictate that a woman's worth is based on her economic earning power [28]; 4) the lack of physician breastfeeding education $[13,16]$ and 5) The mass sexualization of the female breast $[7,16]$.

The Surgeon General [13] has stated that increasing breastfeeding rates is essential, and has urged researchers to improve the public's understanding of the compendious benefits associated with breastfeeding [13]. At the present time, the United States of America has one of the lowest breastfeeding rates in the world, and continues to rank significantly higher than other industrialized nations in terms of morbidity and mortality rates [15]. If we are to see a reduction in overweight and obesity, American's can no longer afford to ignore the protective effects of breastfeeding which have been well documented in the medical literature.

In light of the overwhelming body of scientific evidence that documents the risks associated with formula feeding, the medical profession, parents, researchers, educators, and concerned others must strengthen their advocacy of breastfeeding. We can no longer claim that breastfeeding and formula feeding are equal methods of nutrition, or that health outcomes are the same for breastfed and formula fed populations.

Data confirms that breastfeeding in infancy is associated with a reduced risk of overweight and obesity, type 2 diabetes, elevated blood pressure and cholesterol, and cardiovascular disease $[5,11,12,36]$. While the decrease in caloric intake and an increase in physical activity are the primary environmental protections against overweight and obesity, preventative strategies such as increasing breastfeeding initiation and duration rates are essential if we are to be successful in reversing the overweight and obesity epidemic in future generations [5]. Accordingly, we must address this obesity crisis by making breastfeeding a public health priority, and by uniting forces across disciplines to support a strong and effective public health campaign to increase breastfeeding rates exponentially $[10,11]$.

\section{FUTURE DIRECTIONS}

Extensive epidemiological data demonstrates that exclusive breastfeeding has long term benefits including 
the reduction of obesity and overweight, type 2 diabetes, blood pressure, LDL cholesterol levels, and cardiovascular disease [5,10,15,37]. The inverse relationship between breastfeeding and the above stated risk factors can no longer be ignored. According to decades of published empirical data, breastfeeding significantly impacts health outcomes not only in childhood, but throughout the life-span [14,16,11].

In order to increase breastfeeding rates exponentially, experts have suggested the following:

- Requiring that the formula industry inform the American consumer of the risks associated with their product $[7,16]$.

- Incorporating breastfeeding role models in the familial sphere, the community, and the mass media [7].

- Regulating the advertising of formula, particularly in medical journals and parenting magazines [7].

- Demanding that the economic alliance between the medical community and the formula industry be severed, including a halt to the formula industry's funding of medical research, as well as its practice of giving free samples to new mothers via hospitals or physician offices [28].

- Reassessing America's cultural view of the female breast [16,28].

- Providing mandatory continuing breastfeeding education for practicing physicians [16,17].

- Formally questioning the ethics of medical journals that carry advertisements for formula (a product that is known to increase morbidity and mortality rates) [28].

- Demanding that the public be informed via public service announcements of the protective effects of breastfeeding.

- Requiring physicians to inform their patients that breastfeeding significantly decreases morbidity and mortality rates (including overweight and obesity).

\section{REFERENCES}

[1] Neilsen, S. and Popkin, B. (2003) Patterns and trends in food portion sizes. Journal of the American Medical Association, 289, 450-454. doi:10.1001/jama.289.4.450

[2] Miller, J., Rosenbloom, A. and Silverstein, J. (2004) Childhood obesity. The Journal of Clinical Endocrinology \& Metabolism, 89, 4211-4220. doi:10.1210/jc.2004-0284

[3] Stolzer, J. (2005) ADHD in America: A bioecological analysis. Ethical Human Psychology and Psychiatry, 7, 65-75.

[4] Livingstone, M., Robson, P., Wallace, J. and McKinley, M., (2003) How active are we? Levels of routine physiccal activity in children and adults. Proceedings of the Nutrition Society, 62, 681-701. doi:10.1079/PNS2003291

[5] American Academy of Pediatrics (2003) Policy state- ment: The prevention of pediatric overweight and obesity. Pediatrics, 110, 496-507.

[6] Baumslag, N. and Michels, B. (1995) Milk, money, and madness: The culture and politics of breastfeeding. Bergin and Garvey, London.

[7] Palmer, G. (1991) The politics of breastfeeding. Harper Collins, London.

[8] Margolis, L. (1991) The ethics of accepting gifts from pharmaceutical companies. Pediatrics, 88, 39-54.

[9] Goran, M. (2001) Metabolic precursors and effects of obesity in children: A decade of progress, 1990-1999. American Journal of Clinical Nutrition, 73, 158-171.

[10] Deckelbaum, R. and Williams, C. (2001) Childhood obesity: The health issue. Obesity Research, 9, 239-243. doi:10.1038/oby.2001.125

[11] World Health Organization (2007) Evidence on the long term effects of breastfeeding: Systematic reviews and meta-analyses. Geneva, Switzerland, 1-52.

[12] Freedman, D., Dietz, W., Srinivasan, S. and Berenson, G. (1999) The relation of overweight to cardiovascular risk factors among children and adolescents: The Bogalusa Heart Study. Pediatrics, 103, 1175-1182. doi:10.1542/peds.103.6.1175

[13] United States Department of Health and Human Services (2000) HHS Blueprint for action on breastfeeding. Washington, DC.

[14] American Academy of Pediatrics (2005) Policy Statement: Breastfeeding and the use of human milk. Pediatrics, 115, 496-506. doi:10.1542/peds.2004-2491

[15] World Health Organization (2000) Obesity: Preventing and managing the global epidemic. World Health Organization, Series 894, Geneva, Switzerland.

[16] Stolzer, J. and Hossain, S. (2005) Physician breastfeeding education: A regional assessment. The Female $\mathrm{Pa}$ tient, 30, 59-71.

[17] Stolzer, J. and Zeece, P. (2006) Low income women and physician breastfeeding advice: A regional assessment. Health Education Journal, 65, 158-176. doi:10.1177/001789690606500203

[18] Kramer, M. (1981) Do breastfeeding and delayed introduction of solid foods protect against subsequent obesity? Journal of Pediatrics, 98, 883-887. doi:10.1016/S0022-3476(81)80579-3

[19] Lucas, A., Boyes, S., Bloom, R. and Aynsley-Green, A. (1981) Metabolic and endocrine responses to a milk feed in six-day-old term infants: Differences between breast and cow's milk formula feeding. Acta Paediatra Scandivica, 70, 195-200.

[20] McCance, R. (1962) Food, growth and time. Lancet, 2, 671-676. doi:10.1016/S0140-6736(62)90499-3

[21] Morley, R. and Dwyer, T. (2002) Early exposures and later health and development. Public Health Issues in Infant and Child Nutrition, 48, 257-278.

[22] Waterland, R. and Garza, C. (1999) Potential mechanisms of metabolic imprinting that leads to chronic disease. American Journal of Clinical Nutrition, 69, 179-197.

[23] Dewey, K. (2003) Is breastfeeding protective against childhood obesity? Journal of Human Lactation, 19, 9-18. doi:10.1177/0890334402239730

[24] Stini, W. (1978) Early nutrition, growth, disease and human longevity. Nutrition and Cancer, 1, 31-39. doi:10.1080/01635587809513599 
[25] Balaban, G. and Silva, G. (2004) Protective effect of breastfeeding against childhood obesity. Journal de Pediatria, 80, 419-428.

[26] Hamosh, M. (2001) Bioactive factors in human milk. Pediatric Clinics of North America, 48, 1-19. doi:10.1016/S0031-3955(05)70286-8

[27] Cripps, R., Martin-Gronert, M. and Ozanne, M. (2005) Fetal and perinatal programming of appetite. Clinical Science, 109, 1-11. doi:10.1042/CS20040367

[28] Stuart-Macadam, P. and Dettwyler, K. (1995) Breastfeeding: Biocultural perspectives. Aldine DeGruyter, New York.

[29] Armstrong, J. and Reilly, J. (2002) Breastfeeding and lowering the risk of childhood obesity. Lancet, 359, 2003-2006. doi:10.1016/S0140-6736(02)08837-2

[30] Toschke, A., Vignerova, J., Lhotska, L., Osancova, K., Koletzko, B. and Von Kries, R. (1991) Overweight and obesity in 6 to 14 year old Czech children in 1991: Protective effect of breastfeeding. Journal of Pediatrics, 141, 764-769. doi:10.1067/mpd.2002.128890

[31] Gillman, M., Rifas-Shiman, S., Camargo, C., Berkey, C., Frazier, L,. Rockett, H., Field, A. and Colditz, A. (2001) Risk of overweight among adolescents who were breastfed as infants. Journal of the American Medical Association, 285, 2461-2470. doi:10.1001/jama.285.19.2461

[32] Bergmann, K., Bergmann, R., Von Kries, R., Bohm, O., Richter, R., Dudenhauser, J. and Wahn, U. (2003) Early determinants of child overweight and adiposity in a birth cohort study: Role of breastfeeding. International Journal of Obesity, 27, 162-172. doi:10.1038/sj.ijo.802200

[33] Mayer-Davis, E., Rifas-Shiman, S., Zhou, L., Hu, F., Colditz, G. and Gillman, M. (2006) Breastfeeding and risk for childhood obesity: Does maternal diabetes or obesity status matter? Diabetes Care, 22, 38-51.

[34] Arenz, S., Ruckerl, R. and Von Kries, R. (2004) Breastfeeding and childhood obesity: A systematic review. International Journal of Obesity, 28, 1247-1256. doi:10.1038/sj.ijo.0802758

[35] Rajala, M. and Scherer, P. (2003) Minireview: The adipocyte at the crossroads of energy homeostasis, inflamemation, and atherosclerosis. Endocrinology, 144, 3765-
3785. doi:10.1210/en.2003-0580

[36] Owen, C., Martin, R., Whincup, P., Smith, G. and Cook, D. (2006) Does breastfeeding influence risk of type 2 diabetes in later life? A quantitative analysis of published evidence. American Journal of Clinical Nutrition, 84, 1043-1054.

[37] Ravelli, A. (2000) Infant feeding and adult glucose tolerance, lipid profile, blood pressure, and obesity. Archives of Diseases in Childhood, 82, 248-253.

[38] Martin, R. (2005) Breastfeeding in infancy and blood pressure in later life: Systematic review and meta-analysis. American Journal of Epidemiology, 161, 15-26. doi:10.1093/aje/kwh338

[39] Plagemann, A. and Harder, T. (2005) Breastfeeding and the risk of obesity and related metabolic diseases in the child. Metabolic Syndrome and Related Disorders, 3, 222-229. doi:10.1089/met.2005.3.222

[40] Singhal, A., Cole, T., Fewtrell, M. and Lucas, A. (2004) Breastmilk feeding and lipoprotein profile in adolescents born preterm: Follow-up of a prospective randomized study. The Lancet, 363, 9421-9428. doi:10.1016/S0140-6736(04)16198-9

[41] Davis, P., Dawson, J., Riley, W. and Lauer, R. (2001) Carotid intimal-medical thickness is related to cardiovascular risk factors measured from childhood through middle ages. The Muscatine Study. Circulation, 104, 2815-2819. doi:10.1161/hc4601.099486

[42] Rich-Edwards, J., Stampfer, M., Manson, J., Rosner, B., $\mathrm{Hu}, \mathrm{F}$., Michels, K. and Willet, W. (2004) Breastfeeding during infancy and the risk of cardiovascular disease in adulthood. Epidemiology, 15, 550-556. doi:10.1097/01.ede.0000129513.69321.ba

[43] Owen, C., Whincup, P, Odoki, K., Gilg, J. and Cook, D. (2002) Infant feeding and blood cholesterol: A study of adolescents and a systematic review. Pediatrics, 110, 597-608. doi:10.1542/peds.110.3.597

[44] Tracy, R., Newman, W., Wattigney, W. and Berenson, G. (1995) Risk factors and atherosclerosis in youth autopsy findings of the Bogalusa Heart Study. American Journal of Medical Science, 310, 537-541. doi:10.1097/00000441-199512000-00007 